\title{
EXACT DISTRIBUTION OF THE LARGEST AND SMALLEST EIGENVALUES OF THE RATIO OF TWO ELLIPTICAL WISHART MATRICES
}

\author{
AYA SHINOZAKI and HIROKI HASHIGUCHI \\ Graduate School of Science \\ Tokyo University of Science \\ Japan \\ e-mail: 1413704@ed.tus.ac.jp
}

\begin{abstract}
We discuss a class of generalized Wishart distributions under elliptical models that is called the elliptical Wishart distribution. We derive the distribution of the largest and smallest eigenvalues for the ratio of two sample Wishart matrices under elliptical models. These general results include classical expressions for the Gaussian model. We also discuss a matrix-variate $t$ distribution as a special case.
\end{abstract}

\section{Introduction}

The exact distributions of the largest and smallest eigenvalues of a sample covariance matrix were given respectively by Sugiyama [13] and Khatri [8] under multivariate normality. Khatri [8] also explained the distribution of the smallest eigenvalue for the ratio of sample covariance matrices. The test that uses the ratio as a statistics is called the two 
samples problem. Chikuse [4] discussed the problem with investigation of the asymptotic behaviour of the distribution of eigenvalues of the ratio of the covariance matrices. Sugiura [11,12] studied asymptotic expansions for the distribution of eigenvalues. Fujikoshi [5] discussed them under nonnormality. Hashiguchi et al. [6] derived the density function of the ratio and demonstrated that its cumulative distribution function can be expressed in terms of a Gauss hypergeometric function of a matrix argument.

Recently, discussion under a multivariate normality has been extended to that under elliptically contoured models. Caro-Lopera et al. [1] introduced the configuration densities corresponding to the matrix variate elliptical contoured distributions, including Pearson, Kotz, Bessel and Jensen-logistic distributions. Caro-Lopera et al. [2,3] discussed an extension of a Wishart distribution to the elliptical model in the framework of their earlier work, described in a report by Caro-Lopera et al. [1]. Actually, Caro-Lopera et al. [2] derived the joint distribution of the eigenvalues of two sample covariance matrices and studied the noncentral moments of the modified likelihood ratio statistic under elliptical models. Later, Caro-Lopera et al. [3] derived the joint distribution of the eigenvalues of a sample covariance matrix and the distributions of the largest and smallest eigenvalues. Shinozaki et al. [10] provided an alternative proof of the derivation of the distribution of the largest eigenvalue, in contrast to the result presented by Caro-Lopera et al. [3]. They also conducted numerical experiments under a matrix-variate $t$ distribution.

In this paper, we discuss the two samples problem under the elliptically contoured model with analogy to Hashiguchi et al. [6]. In the remainder of this report of our work, we introduce the elliptical Wishart distribution and some fundamental results. In Section 2, we derive the distribution function of the ratio of elliptical Wishart matrices and its largest and smallest eigenvalues. Conclusions and future work are described in Section 3. 
The elliptical Wishart distribution is written as $\mathcal{E W}_{m}(n, \Sigma, h)$. Its density function of $W$ is given as

$$
\frac{\pi^{m n / 2}}{\Gamma_{m}\left(\frac{1}{2} n\right)|\Sigma|^{n / 2}}|W|^{(n-m-1) / 2} h\left(\operatorname{tr} \Sigma^{-1} W\right),
$$

where $|W|$ is the determinant of $W, \operatorname{tr} W$ denotes the trace of $W, \operatorname{etr}(W)=$ $\exp (\operatorname{tr} W), \Gamma_{m}(a)=\pi^{m(m-1) / 4} \prod_{i=1}^{m} \Gamma(a-(i-1) / 2)$, with $\Re(a)>(m-1) / 2$, and $\Gamma(a)$ is the ordinary gamma function.

James [7] provided the definition and properties of a zonal polynomial in multivariate analysis. Let $\mathcal{C}_{\kappa}(X)$ be the zonal polynomial with a symmetric matrix $X$ indexed by a partition of $k$ into not more than $m$ parts $\kappa=\left(\kappa_{1}, \ldots, \kappa_{m}\right)$, where $\kappa_{1} \geq \cdots \geq \kappa_{m} \geq 0$ and $\sum_{i=1}^{m} \kappa_{i}=k$. The sum $\sum_{\kappa}$ runs over all partitions of $k$.

The Pochhammer symbol for a partition $\kappa=\left(\kappa_{1}, \ldots, \kappa_{m}\right)$ is defined as $(\alpha)_{\kappa}=\prod_{i=1}^{m}(\alpha-(i-1) / 2)_{\kappa_{i}}$, where $(\alpha)_{t}=\alpha(\alpha+1) \cdots(\alpha+t-1)$ and $(\alpha)_{0}=1$. The following three lemmas related to the zonal polynomial are useful to derive our main theorem in Section 2.

Lemma 1 (Caro-Lopera et al. [1]). Let $Z$ be a complex symmetric $m \times m$ matrix with $\Re(Z)>0$ and let $Y$ be a symmetric $m \times m$ matrix. If $k$ is a nonnegative integer and $\kappa$ is a partition of $k$, then, for $\Re(a)>(m-1) / 2$,

$$
\int_{X>0} h(\operatorname{tr} X Z)|X|^{a-(m+1) / 2} \mathcal{C}_{\kappa}(X Y)(d X)=\frac{|Z|^{-a}(a)_{\kappa} \Gamma_{m}(a) \mathcal{C}_{\kappa}\left(Y Z^{-1}\right)}{\Gamma(m a+k)} H_{k}
$$


where

$$
H_{k}=\int_{0}^{\infty} h(w) w^{m a+k-a} d w<\infty
$$

An important analogue of the beta function integral is given in the following lemma.

Lemma 2 (Muirhead [9]). If $Y$ is a symmetric $m \times m$ matrix, then

$$
\int_{O<X<I_{m}}|X|^{a-(m+1) / 2}\left|I_{m}-X\right|^{b-(m+1) / 2} \mathcal{C}_{\kappa}(X Y)(d X)=\frac{(a)_{\kappa}}{(a+b)_{\kappa}} \frac{\Gamma_{m}(a) \Gamma_{m}(b)}{\Gamma_{m}(a+b)} C_{\kappa}(Y),
$$

for $\Re(a)>(m-1) / 2, \Re(b)>(m-1) / 2$.

Lemma 3 (Sugiyama [13]). Let $\mathbf{X}_{1}=\operatorname{diag}\left(1, x_{2}, \ldots, x_{m}\right), \mathbf{X}_{2}=$ $\operatorname{diag}\left(x_{2}, \ldots, x_{m}\right)$, where $x_{2}>\cdots>x_{m}>0$. Then it holds that

$$
\begin{gathered}
\int_{1>x_{2}>\cdots>x_{m}>0}\left|\mathbf{X}_{2}\right|^{t-(m+1) / 2} \mathcal{C}_{\kappa}\left(\mathbf{X}_{1}\right) \prod_{i=2}^{m}\left(1-x_{i}\right) \prod_{i<j}\left(x_{i}-x_{j}\right) \prod_{i=2}^{m} d x_{i} \\
=(m t+k)\left(\Gamma_{m}(m / 2) / \pi^{m^{2} / 2}\right) \frac{\Gamma_{m}(t)(t)_{\kappa} \Gamma_{m}((m+1) / 2)}{\Gamma_{m}(t+(m+1) / 2)(t+(m+1) / 2)_{\kappa}} \mathcal{C}_{\kappa}\left(\mathbf{I}_{m}\right) .
\end{gathered}
$$

We consider that $W_{1}$ and $W_{2}$ are two independent elliptical Wishart matrices, i.e., $W_{1} \sim \mathcal{E W}_{m}\left(n_{1}, \Sigma_{1}, h_{1}\right)$ and $W_{2} \sim \mathcal{E} \mathcal{W}_{m}\left(n_{2}, \Sigma_{2}, h_{2}\right)$, where $n_{1}, n_{2} \geq m$ and $\Sigma_{1}, \Sigma_{2}$ are positive definite. Roy's test statistics use the largest or smallest eigenvalue of $W_{1} W_{2}^{-1}$ for testing the equality of covariance matrices as $H_{0}: \Sigma_{1}=\Sigma_{2}$.

\section{Distribution of the Largest and Smallest Eigenvalues of Ratio for two Elliptical Wishart Matrices}

This section presents the distribution and density function of the largest and smallest eigenvalues of $W_{1} W_{2}^{-1}$. Let $\ell_{1}, \ldots, \ell_{m}$ be the 
eigenvalues of $W_{1} W_{2}^{-1}$, where $\ell_{1}>\cdots>\ell_{m}>0$. This section presents the distribution and density function of the largest eigenvalue $\ell_{1}$ of $W_{1} W_{2}^{-1}$.

Theorem 1. Letting $W_{1} \sim \mathcal{E W}_{m}\left(n_{1}, \Sigma_{1}, h_{1}\right)$ and $W_{2} \sim \mathcal{E W}_{m}\left(n_{2}\right.$, $\left.\Sigma_{2}, h_{2}\right)$, where $n_{1}, n_{2} \geq m$ and $\Sigma_{1}, \Sigma_{2}$ are positive definite, then the distribution function on $W_{1} W_{2}^{-1}$ is given as

$$
\begin{aligned}
& \operatorname{Pr}\left(W_{1} W_{2}^{-1}<\Omega\right)=\frac{\pi^{\left(n_{1}+n_{2}\right) m / 2} \Gamma_{m}\left(\left(n_{1}+n_{2}\right) / 2\right) \Gamma_{m}((m+1) / 2)}{\Gamma_{m}\left(n_{2} / 2\right) \Gamma_{m}\left(\left(n_{1}+m+1\right) / 2\right)}\left|\Sigma^{-1} \Omega\right|^{n_{1} / 2} \\
& \quad \times \sum_{k=0}^{\infty} \frac{h_{1}^{(k)}(0) H_{2 ; k}}{k ! \Gamma\left(\left(n_{1}+n_{2}\right) m / 2+k\right)} \sum_{\kappa} \frac{\left(\left(n_{1}+n_{2}\right) / 2\right)_{\kappa}\left(n_{1} / 2\right)_{\kappa}}{\left(\left(n_{1}+m+1\right) / 2\right)_{\kappa}} \mathcal{C}_{\kappa}\left(\Sigma^{-1} \Omega\right),
\end{aligned}
$$

where $h^{(k)}(a)=d^{k} h(u) /\left.d u^{k}\right|_{u=a}, \Sigma=\Sigma_{1} \Sigma_{2}^{-1}$,

$$
H_{2 ; k}=\int_{0}^{\infty} h_{2}(w) w^{m\left(n_{1}+n_{2}\right) / 2+k-1} d w
$$

and $H_{2 ; k}<\infty$.

Proof. This proof is presented in a similar way to that of Theorem 2 in Hashiguchi et al. [6]. We assume $\Sigma_{1}=\Sigma, \Sigma_{2}=I_{m}$ without loss of generality. The joint density of $\left(W_{1}, W_{2}\right)$ is given as

$$
\begin{aligned}
f\left(W_{1}, W_{2}\right)=\frac{\pi^{\left(n_{1}+n_{2}\right) m / 2}}{\Gamma_{m}\left(n_{1} / 2\right) \Gamma_{m}\left(n_{2} / 2\right)|\Sigma|^{n_{2} / 2}}\left|W_{1}\right|^{\left(n_{1}-m-1\right) / 2}\left|W_{2}\right|^{\left(n_{2}-m-1\right) / 2} & \\
& \times h_{1}\left(\operatorname{tr} \Sigma^{-1} W_{1}\right) h_{2}\left(\operatorname{tr} W_{2}\right) .
\end{aligned}
$$

Let $\quad F=W_{1}^{1 / 2} W_{2}^{-1} W_{1}^{1 / 2}$ and $W_{2}=W_{1}^{1 / 2} F^{-1} W_{1}^{1 / 2}$. Then, using the Jacobian of the transformation $\left(W_{1}, W_{2}\right) \rightarrow\left(W_{1}, F\right)$ as

$$
\left(d W_{2}\right)=\left|W_{1}\right|^{(m+1) / 2}\left|F^{-1}\right|^{m+1}(d F),
$$


and the Maclaurin expansion for $h(\cdot)$ as

$$
h_{1}\left(\operatorname{tr} \Sigma^{-1} W_{1}\right)=\sum_{k=0}^{\infty} \frac{h_{1}^{(k)}(0)}{k !}\left(\operatorname{tr} \Sigma^{-1} W_{1}\right)^{k}=\sum_{k=0}^{\infty} \frac{h_{1}^{(k)}(0)}{k !} \sum_{\kappa} C_{\kappa}\left(\Sigma^{-1} W_{1}\right),
$$

the joint density of $\left(W_{1}, F\right)$ is given as

$$
\begin{aligned}
f\left(W_{1}, F\right)= & \frac{\pi^{\left(n_{1}+n_{2}\right) m / 2}}{\Gamma_{m}\left(n_{1} / 2\right) \Gamma_{m}\left(n_{2} / 2\right)|\Sigma|^{n_{1} / 2}}\left|W_{1}\right|^{\left(n_{1}+n_{2}-(m+1)\right) / 2}|F|^{\left(-n_{2}-m-1\right) / 2} \\
& \times h_{2}\left(\operatorname{tr} W_{1} F^{-1}\right) \sum_{k=0}^{\infty} \frac{h_{1}^{(k)}(0)}{k !} \sum_{\kappa} C_{\kappa}\left(\Sigma^{-1} W_{1}\right) .
\end{aligned}
$$

From Lemma 1, the density of $F$ is given as

$$
\begin{aligned}
f(F)= & \int_{W_{1}>O} f\left(W_{1}, F\right)\left(d W_{1}\right) \\
= & \frac{\pi^{\left(n_{1}+n_{2}\right) m / 2} \Gamma_{m}\left(\left(n_{1}+n_{2}\right) / 2\right)}{\Gamma_{m}\left(n_{1} / 2\right) \Gamma_{m}\left(n_{2} / 2\right)|\Sigma|^{n_{1} / 2}}|F|^{\left(n_{1}-m-1\right) / 2} \\
& \times \sum_{k=0}^{\infty} \frac{h_{1}^{(k)}(0) H_{2 ; k}}{k ! \Gamma\left(m\left(n_{1}+n_{2}\right) / 2+k\right)} \sum_{\kappa}\left(\left(n_{1}+n_{2}\right) / 2\right)_{\kappa} \mathcal{C}_{\kappa}\left(\Sigma^{-1} F\right),
\end{aligned}
$$

where $H_{2 ; k}$ is given in (2). Next, the probability of $F<\Omega$ is given as

$$
\begin{aligned}
& \operatorname{Pr}(F<\Omega)=\int_{F<\Omega} f(F)(d F) \\
& =\frac{\pi^{\left(n_{1}+n_{2}\right) m / 2} \Gamma_{m}\left(\left(n_{1}+n_{2}\right) / 2\right)}{\Gamma_{m}\left(n_{1} / 2\right) \Gamma_{m}\left(n_{2} / 2\right)|\Sigma|^{n_{1} / 2}} \sum_{k=0}^{\infty} \frac{h_{1}^{(k)}(0)}{k !} \frac{H_{2 ; k}}{\Gamma\left(\left(n_{1}+n_{2}\right) m / 2+k\right)} \sum_{\kappa}\left(\left(n_{1}+n_{2}\right) / 2\right)_{\kappa} \\
& \quad \times \int_{O<F<\Omega}|F|^{\left(n_{1}-m-1\right) / 2} \mathcal{C}_{\kappa}\left(\Sigma^{-1} F\right)(d F) .
\end{aligned}
$$


Substituting as $F=\Omega^{1 / 2} X \Omega^{1 / 2}$, the integral is reduced over $O<X<I$ with $(d F)=\left|\Omega^{1 / 2}\right|^{m+1}(d X)$. From Lemma 2, we have

$$
\begin{aligned}
& \int_{O<F<\Omega}|F|^{\left(n_{1}-m-1\right) / 2} \mathcal{C}_{\kappa}\left(\Sigma^{-1} F\right)(d F) \\
= & \int_{O<X<I}|\Omega|^{\left(n_{1}-(m+1)\right) / 2}|X|^{\left(n_{1}-(m+1)\right) / 2} \mathcal{C}_{\kappa}\left(\Sigma^{-1} \Omega^{1 / 2} X \Omega^{1 / 2}\right)\left|\Omega^{1 / 2}\right|^{m+1}(d X) \\
= & |\Omega|^{n_{1} / 2} \frac{\left(n_{1} / 2\right)_{\kappa}}{\left(\left(n_{1}+m+1\right) / 2\right)_{\kappa}} \frac{\Gamma_{m}\left(n_{1} / 2\right) \Gamma_{m}((m+1) / 2)}{\Gamma_{m}\left(\left(n_{1}+m+1\right) / 2\right)} \mathcal{C}_{\kappa}\left(\Sigma^{-1} \Omega\right) .
\end{aligned}
$$

As a result, we have

$$
\begin{aligned}
& \operatorname{Pr}(F<\Omega)=\frac{\pi^{\left(n_{1}+n_{2}\right) m / 2} \Gamma_{m}\left(\left(n_{1}+n_{2}\right) / 2\right) \Gamma_{m}((m+1) / 2)}{\Gamma_{m}\left(n_{2} / 2\right) \Gamma_{m}\left(n_{1} / 2+(m+1) / 2\right)}\left|\Sigma^{-1} \Omega\right|^{n_{1} / 2} \\
& \quad \times \sum_{k=0}^{\infty} \frac{h_{1}^{(k)}(0)}{k !} \frac{H_{2 ; k}}{\Gamma\left(\left(n_{1}+n_{2}\right) m / 2+k\right)} \sum_{\kappa} \frac{\left(\left(n_{1}+n_{2}\right) / 2\right)_{\kappa}\left(n_{1} / 2\right)_{\kappa}}{\left(n_{1} / 2+(m+1) / 2\right)_{\kappa}} C_{\kappa}\left(\Sigma^{-1} \Omega\right) .
\end{aligned}
$$

In fact, the distribution $\mathcal{E}_{n \times m}(M, \Omega \otimes \Sigma, h)$ includes the matrix variate normal distribution $\mathcal{N}_{n \times m}(M, \Omega \otimes \Sigma)$, where $n \geq m$, if $h(y)=\exp (-y / 2) /(2 \pi)^{m n / 2}$. Thereby, if $X \sim \mathcal{N}_{n \times m}\left(O, I_{n} \otimes \Sigma\right)$, i.e., if the row vectors of $X$ are mutually independent and identically distributed as $\mathcal{N}_{m}(O, \Sigma)$, then $W=X^{\top} X$ is distributed as the Wishart distribution $\mathcal{W}_{m}(n, \Sigma)$. The generator function and its $k$-th derivative for the Gaussian case are given respectively as

$$
h_{1}(w)=\frac{1}{(2 \pi)^{m n_{1} / 2}} e^{-w / 2} \text { and } h_{1}^{(k)}(0)=\frac{1}{(2 \pi)^{m n_{1} / 2}}\left(-\frac{1}{2}\right)^{k} \text {. }
$$


Therefore, from Theorem 1 and

$$
H_{2 ; k}=\int_{0}^{\infty}\left(\frac{1}{2 \pi}\right)^{m n_{2} / 2} e^{-w / 2} w^{m\left(n_{1}+n_{2}\right) / 2+k-1} d w=\frac{1}{(2 \pi)^{m n_{2} / 2}} \frac{\Gamma\left(m\left(n_{1}+n_{2}\right) / 2+k\right)}{(1 / 2)^{m\left(n_{1}+n_{2}\right) / 2+k}},
$$

the distribution function of $W_{1} W_{2}^{-1}$ is given as

$$
\begin{aligned}
\operatorname{Pr}\left(W_{1} W_{2}^{-1}<\Omega\right)= & \frac{\Gamma_{m}\left(\left(n_{1}+n_{2}\right) / 2\right) \Gamma_{m}((m+1) / 2)}{\Gamma_{m}\left(n_{2} / 2\right) \Gamma_{m}\left(n_{1} / 2+(m+1) / 2\right)}\left|\Sigma^{-1} \Omega\right|^{n_{1} / 2} \\
& \times{ }_{2} F_{1}\left(\frac{n_{1}+n_{2}}{2}, \frac{n_{1}}{2} ; \frac{n_{1}+m+1}{2} ;-\Sigma^{-1} \Omega\right) .
\end{aligned}
$$

Corollary 1. Substitute $\Omega=x I$ for (1) in Theorem 1, the distribution function of the largest eigenvalue $\ell_{1}$ of $W_{1} W_{2}^{-1}$ is given as

$$
\begin{aligned}
\operatorname{Pr}\left(\ell_{1}\right. & <x)=\frac{\pi^{\left(n_{1}+n_{2}\right) m / 2} \Gamma_{m}\left(\left(n_{1}+n_{2}\right) / 2\right) \Gamma_{m}((m+1) / 2)}{\Gamma_{m}\left(n_{2} / 2\right) \Gamma_{m}\left(\left(n_{1}+m+1\right) / 2\right)}\left|x \Sigma^{-1}\right|^{n_{1} / 2} \\
& \times \sum_{k=0}^{\infty} \frac{h_{1}^{(k)}(0) H_{2 ; k}}{k ! \Gamma\left(\left(n_{1}+n_{2}\right) m / 2+k\right)} \sum_{\kappa} \frac{\left(\left(n_{1}+n_{2}\right) / 2\right)_{\kappa}\left(n_{1} / 2\right)_{\kappa}}{\left(\left(n_{1}+m+1\right) / 2\right)_{\kappa}} C_{\kappa}\left(x \Sigma^{-1}\right) .
\end{aligned}
$$

The above (4) is derived from the multiple integral of the joint density of $\ell_{1}, \ldots, \ell_{m}$. The joint density of $\ell_{1}, \ldots, \ell_{m}$ was given by Caro-Lopera et al. [2] as

$$
\begin{aligned}
& \frac{\pi^{m\left(m+n_{1} n_{2}\right) / 2} \Gamma_{m}\left(\left(n_{1}+n_{2}\right) / 2\right)}{\Gamma_{m}(m / 2) \Gamma_{m}\left(n_{1} / 2\right) \Gamma_{m}\left(n_{2} / 2\right)}\left|\Sigma^{-1}\right|^{n_{1} / 2} \prod_{i=1}^{m} \ell_{i}^{\left(n_{1}-m-1\right) / 2} \prod_{i<j}^{m}\left(\ell_{i}-\ell_{j}\right) \\
& \quad \times \sum_{k=0}^{\infty} \frac{h_{1}^{(k)}(0) H_{2 ; k}}{k ! \Gamma\left(\left(n_{1}+n_{2}\right) m / 2+k\right)} \sum_{\kappa}\left(\left(n_{1}+n_{2}\right) / 2\right)_{\kappa} \frac{\mathcal{C}_{\kappa}\left(\Sigma^{-1}\right) \mathcal{C}_{\kappa}(L)}{\mathcal{C}_{\kappa}\left(I_{m}\right)},
\end{aligned}
$$

where $L=\operatorname{diag}\left(\ell_{1}, \ldots, \ell_{m}\right)$ and $\Sigma=\Sigma_{1} \Sigma_{2}^{-1}$. In a similar mode of Shinozaki et al. [10], the distribution of the largest eigenvalue can be derived from the multiple integral of (5). Translating $\ell_{i}$ to $x_{i}=\ell_{i} / \ell_{1}$ 
for $i=2, \ldots, m$ and putting $X_{1}=\operatorname{diag}\left(1, x_{2}, \ldots, x_{m}\right)$ and $X_{2}=\operatorname{diag}$ $\left(x_{2}, \ldots, x_{m}\right)$, then (5) is can be written as

$$
\begin{aligned}
& \frac{\pi^{m\left(m+n_{1} n_{2}\right) / 2} \Gamma_{m}\left(\left(n_{1}+n_{2}\right) / 2\right)}{\Gamma_{m}(m / 2) \Gamma_{m}\left(n_{1} / 2\right) \Gamma_{m}\left(n_{2} / 2\right)}\left|\Sigma^{-1}\right|^{n_{1} / 2} \\
& \quad \times \ell_{1}^{m\left(n_{1}-2\right) / 2+k}\left|X_{2}\right|^{\left(n_{1}-m-1\right) / 2} \prod_{i=2}^{m}\left(1-x_{i}\right) \prod_{2 \leq i<j}^{m}\left(x_{i}-x_{j}\right) \\
& \quad \times \sum_{k=0}^{\infty} \frac{h_{1}^{(k)}(0) H_{2 ; k}}{k ! \Gamma\left(\left(n_{1}+n_{2}\right) m / 2+k\right)} \sum_{\kappa}\left(\left(n_{1}+n_{2}\right) / 2\right)_{\kappa} \frac{\mathcal{C}_{\kappa}\left(\Sigma^{-1}\right) \mathcal{C}_{\kappa}\left(X_{1}\right)}{\mathcal{C}_{\kappa}\left(I_{m}\right)} .
\end{aligned}
$$

An alternative proof of Corollary 1 is completed from Lemma 3.

We now consider the distribution of the smallest eigenvalue $\ell_{m}$ of $W_{1} W_{2}^{-1}$. The event $\left\{\ell_{m}>x\right\}$ are equivalent to the one $\left\{W_{1} W_{2}^{-1}>x I_{m}\right\}$. Therefore, $\operatorname{Pr}\left(\ell_{m}>x\right)$ is derived from $\operatorname{Pr}\left(\ell_{1}<x\right)$ given by (4) by replacing $n_{1}, n_{2}, x, \Sigma_{1}, \Sigma_{2}, h_{1}$ and $h_{2}$ by $n_{2}, n_{1}, x^{-1}, \Sigma_{2}, \Sigma_{1}, h_{2}$ and $h_{1}$, respectively. This replacement method was described by Chikuse [4] for the Gaussian case.

Corollary 2. The upper probability of the smallest eigenvalue $\ell_{m}$ of $W_{1} W_{2}^{-1}$ is given as

$$
\begin{aligned}
\operatorname{Pr}\left(\ell_{m}\right. & >x)=\frac{\pi^{\left(n_{1}+n_{2}\right) m / 2} \Gamma_{m}\left(\left(n_{1}+n_{2}\right) / 2\right) \Gamma_{m}((m+1) / 2)}{\Gamma_{m}\left(n_{1} / 2\right) \Gamma_{m}\left(\left(n_{2}+m+1\right) / 2\right)}\left|x^{-1} \Sigma^{-1}\right|^{n_{2} / 2} \\
& \times \sum_{k=0}^{\infty} \frac{h_{2}^{(k)}(0) H_{1 ; k}}{k ! \Gamma\left(\left(n_{1}+n_{2}\right) m / 2+k\right)} \sum_{\kappa} \frac{\left(\left(n_{1}+n_{2}\right) / 2\right)_{\kappa}\left(n_{2} / 2\right)_{\kappa}}{\left(\left(n_{2}+m+1\right) / 2\right)_{\kappa}} C_{\kappa}\left(x^{-1} \Sigma^{-1}\right),
\end{aligned}
$$

where $\Sigma=\Sigma_{2} \Sigma_{1}^{-1}$ and $H_{1 ; k}=\int_{0}^{\infty} h_{1}(w) w^{m\left(n_{1}+n_{2}\right) / 2+k-1} d w$ with $H_{1 ; k}<\infty$. 
As an example, the functions $h(y), h_{1}^{(k)}(0)$ and $H_{2 ; k}$ for a matrixvariate $t$ distribution are given by

$$
\begin{gathered}
h(w)=\frac{\Gamma((m n+\rho) / 2)}{(\pi \rho)^{m n / 2} \Gamma(\rho / 2)}(1+w / \rho)^{-\left(m n_{2}+\rho\right) / 2}, \\
h_{1}^{(k)}(0)=\frac{\Gamma\left(\left(m n_{1}+\rho_{1}\right) / 2\right)(-1)^{k}\left(\left(m n_{1}+\rho_{1}\right) / 2\right)_{k}}{\left(\pi \rho_{1}\right)^{m n_{1} / 2} \Gamma\left(\rho_{1} / 2\right) \rho_{1}^{k}},
\end{gathered}
$$

and

$$
H_{2 ; k}=\frac{\rho_{2}^{m n_{1} / 2+k} \Gamma\left(m\left(n_{1}+n_{2}\right) / 2+k\right) \Gamma\left(\left(\rho_{2}-m n_{1}\right) / 2-k\right)}{\pi^{m n_{2} / 2} \Gamma\left(\rho_{2} / 2\right)},
$$

respectively. The distribution function of the largest eigenvalue $\ell_{1}$ of $W_{1} W_{2}^{-1}$ under a matrix-variate $t$ distribution is given as

$$
\begin{aligned}
& \operatorname{Pr}\left(\ell_{1}<x\right)=\frac{\Gamma_{m}\left(\left(n_{1}+n_{2}\right) / 2\right) \Gamma_{m}((m+1) / 2) \Gamma\left(\left(m n_{1}+\rho_{1}\right) / 2\right)}{\Gamma_{m}\left(n_{2} / 2\right) \Gamma_{m}\left(\left(n_{1}+m+1\right) / 2\right) \Gamma\left(\rho_{1} / 2\right) \Gamma\left(\rho_{2} / 2\right)}\left|x \Sigma^{-1}\right|^{n_{1} / 2} \\
& \times \sum_{k=0}^{\left[\rho_{2} / 2-m n_{1} / 2\right]} \frac{(-1)^{k}\left(\left(m n_{1}+\rho_{1}\right) / 2\right)_{k} \Gamma\left(\left(-m n_{1}+\rho_{2}\right) / 2-k\right)}{k !}\left(\frac{\rho_{2}}{\rho_{1}}\right)^{m n_{1} / 2+k} \\
& \quad \times \sum_{\kappa} \frac{\left(\left(n_{1}+n_{2}\right) / 2\right)_{\kappa}\left(n_{1} / 2\right)_{\kappa}}{\left(\left(n_{1}+m+1\right) / 2\right)_{\kappa}} \mathcal{C}_{\kappa}\left(x \Sigma^{-1}\right)
\end{aligned}
$$

\section{Conclusion}

As described herein, we provide proof of the derivation of the largest and smallest eigenvalue distributions under elliptically contoured distributions. A matrix-variate $t$ distribution is given as an example. However, its numerical computation has not been completed yet. We tried the numerical computation under matrix-variate $t$ distribution in a similar mode to that used by Shinozaki et al. [10] but it could not be done well. This is reason why (6) includes an alternating series. We think that 
it is necessary to replace from the alternating series to the positive term series by, e.g., Euler relation of a derivation of the positive term series. That and the numerical computation are left as subjects for future work.

\section{Acknowledgements}

The authors would like to thank the Editor-in-Chief and anonymous referees for careful reading and helpful suggestion. This work was supported by JSPS KAKENHI Grant Numbers JP15K00051, JP18K03428.

\section{References}

[1] F. J. Caro-Lopera, J. A. Díaz-García and G. González-Farías, Noncentral elliptical configuration density, Journal of Multivariate Analysis 101(1) (2010), 32-43.

DOI: https://doi.org/10.1016/j.jmva.2009.03.004

[2] F. J. Caro-Lopera, G. González-Farías and N. Balakrishnan, On generalized Wishart distributions-I: Likelihood ratio test for homogeneity of covariance matrices, Sankhyā: The Indian Journal of Statistics, Series A 76(2) (2014), 179-194.

[3] F. J. Caro-Lopera, G. González-Farías and N. Balakrishnan, Matrix-variate distribution theory under elliptical models-4: Joint distribution of latent roots of covariance matrix and the largest and smallest latent roots, Journal of Multivariate Analysis 145 (2016), 224-235.

DOI: https://doi.org/10.1016/j.jmva.2015.12.012

[4] Y. Chikuse, Asymptotic expansions for the joint and marginal distributions of the latent roots of $S_{1} S_{2}^{-1}$, Annals of the Institute of Statistical Mathematics 29(2) (1977), 221-233.

[5] Y. Fujikoshi, Asymptotic expansions for the distributions of the sample roots under nonnormality, Biometrika 67(1) (1980), 45-51.

DOI: https://doi.org/10.1093/biomet/67.1.45

[6] H. Hashiguchi, N. Takayama and A. Takemura, Distribution of the ratio of two Wishart matrices and cumulative probability evaluation by the holonomic gradient method, Journal of Multivariate Analysis 165 (2018), 270-278.

DOI: https://doi.org/10.1016/j.jmva.2018.01.002

[7] A. T. James, Distribution of matrix variates and latent roots derived from normal samples, Annals of Mathematical Statistics 35(2) (1964), 475-501. 
[8] C. G. Khatri, On the exact finite series distribution of the smallest or the largest root of matrices in three situations, Journal of Multivariate Analysis 2(2) (1972), 201-207.

DOI: https://doi.org/10.1016/0047-259X(72)90027-9

[9] R. J. Muirhead, Aspects of Multivariate Statistical Theory, John Wiley \& Sons, New York, 1982.

[10] A. Shinozaki, H. Hashiguchi and T. Iwashita, Distribution of the largest eigenvalue of an elliptical Wishart matrix and its simulation, Journal of the Japanese Society of Computational Statistics 30(2) (2018), 1-12.

DOI: https://doi.org/10.5183/jjscs.1708001_244

[11] N. Sugiura, Derivatives of the characteristic root of a synmetric or a Hermitian matrix with two applications in multivariate analysis, Communications in Statistics 1(5) (1973), 393-417.

DOI: https://doi.org/10.1080/03610927308827036

[12] N. Sugiura, Asymptotic expansion of the distributions of the latent roots and the latent vector of the Wishart and multivariate $F$ matrices, Journal of Multivariate Analysis 6(4) (1976), 500-525.

DOI: https://doi.org/10.1016/0047-259X(76)90002-6

[13] T. Sugiyama, On the distribution of the largest latent root of the covariance matrix, Annals of Mathematical Statistics 38(4) (1967), 1148-1151. 